\title{
Abuso del móvil en estudiantes universitarios y perfiles de victimización y agresión
}

\section{Mobile Abuse in University Students and Profiles of Victimization and Aggression}

\author{
María Isabel Polo del Río*, Santiago Mendo lázaro*, \\ Benito León del Barco*, Elena Felipe Castaño*. \\ *Departamento de Psicología y Antropología de la Universidad de Extremadura.
}

\section{Resumen}

La gran mayoría de jóvenes disponen de teléfono móvil, convirtiéndose en un objeto imprescindible en su vida, que ha desplazado los espacios de socialización tradicionales por espacios virtuales. Es utilizado por ellos, durante muchas horas, en detrimento de su funcionamiento psicológico y social, mostrando mayor vulnerabilidad a su uso abusivo o excesivo, y mayor propensión a convertirse en un uso problemático o adictivo. En este trabajo se pretende estudiar las repercusiones sociales, personales y comunicacionales del abuso del móvil de los estudiantes universitarios, y profundizar en los diferentes perfiles del ciberacoso, analizando quién presenta más problemas personales y sociales con el uso del móvil: ¿víctimas o agresores? También si el número de horas de uso del móvil tiene un efecto sobre dichos problemas. La muestra (1200 estudiantes) fue seleccionada mediante muestreo polietápico por conglomerados de entre las distintas Facultades de la Universidad de Extremadura. Los datos fueron obtenidos a través de las Escalas de Victimización (CYB-VIC) y Agresión (CYB-AGRES) a través del Teléfono Móvil y el Cuestionario de Experiencias relacionadas con el Móvil (CERM). Los resultados muestran que el uso abusivo del móvil genera conflictos en los jóvenes de ambos sexos; aunque las chicas manifiestan más problemas comunicacionales y emocionales que los chicos. Además, la edad, el campo de conocimiento, el perfil víctima/ agresor y las horas de uso del móvil son variables determinantes sobre los conflictos comunicacionales y emocionales derivados del uso abusivo del móvil.

Palabras clave: Abuso del móvil; Perfiles de ciberacoso; Agresor; víctima; Estudiantes universitarios.

\begin{abstract}
The vast majority of young people have mobile phones. This has become a must-have item in their lives, with traditional socialization spaces displaced by virtual ones. They use their mobile phones for many hours a day, to the detriment of their psychological and social functioning, showing greater vulnerability to abusive or excessive use, and more likely to become problematic or addicted users. This paper aims to study the impact of mobile phone abuse in a sample of college students, assessing the social, personal, and communicational realms and deepening understanding of the different cyberbullying profiles, analyzing who has more personal and social problems using mobiles: victims or aggressors. Whether the number of hours of mobile phone use has an effect on these problems will also be explored. The sample (1,200 students) was selected by multistage cluster sampling among the faculties of the University of Extremadura. Data were obtained through Victimization (CYB-VIC) and Aggression (CYBAGRES) through the mobile phone scales, and the Questionnaire of Experiences related to Mobile (CERM). The results show that mobile phone abuse generates conflicts in young people of both sexes, although girls have more communication and emotional problems than boys. In addition, age, field of knowledge, victim/aggressor profile, and hours of mobile phone use are crucial variables in the communication and emotional conflicts arising from the misuse of mobile.
\end{abstract}

Keywords: Abuse mobile; Profiles ciberacoso; Aggressor; Victim and university students. 


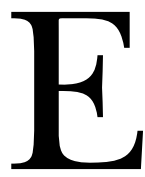
1 uso de las Tecnologías de la Información y las Comunicaciones (TIC) es inherente a las sociedades modernas. Cada vez son mayores las posibilidades de acceso a dichas herramientas y su uso no está exento de problemas. Entre las TIC, el teléfono móvil (teléfono inteligente o Smartphone) es la tecnología más popular. El uso intensivo de los Smartphone ha originado preocupación a investigadores e instituciones (Gómez, Rial, Braña, Varela y Barreiro, 2014). Si bien su uso no supone en sí mismo un problema, sí lo es la relación problemática que se establece con él (Chóliz, 2010; Echeburúa, Labrador y Becoña, 2009), llegando a condicionar las relaciones sociales cuando se utiliza un elevado número de horas al día o de forma descontrolada, (Bianchi y Phillips, 2005; Kamibeppu y Sugiura, 2005).

No existe acuerdo acerca de la frontera entre un uso excesivo y un uso problemático o patológico, dado que el término "adicción" a las TIC aún no ha sido reconocido oficialmente por organismos internacionales, como la American Psyquiatric Association (APA) o la Organización Mundial de la Salud (Labrador, Villadangos, Crespo y Becoña, 2013; López-Fernández, Honrubia-Serrano y Freixa-Blanxart, 2012). Sin embargo, existen evidencias -especialmente en adolescentes y jóvenes-de la aparición de problemas comportamentales, afectivos y sociales relacionados con el uso del teléfono móvil (Pedrero, Rodríguez y Ruiz, 2012).

Son los estudiantes universitarios junto con la población adolescente, los grupos de edad considerados de mayor riesgo (Weare, 2004), el móvil tiene una relevancia mucho mayor para estos grupos, que para poblaciones de otras edades (Kubey, Lavin y Barrow, 2001; Morahan-Martin y Schumacher, 2000; Treuer, Fabián y Friredi, 2001), pues la gran mayoría de jóvenes disponen de uno, transformándose en un objeto imprescindible en su vida y en su tiempo de ocio. El móvil es fundamentalmente una herramienta relacional (Aguado y Martínez, 2006) que proporciona los procesos necesarios de comunicación y socialización (Ellwood-Clayton, 2003; INE, 2014; Taylor y Harper, 2003), y que ha desplazado los espacios de socialización tradicionales, dando lugar a espacios virtuales, con una sustancial función social (Díaz-Gandasegui, 2011). La conexión permanente, parece satisfacer la gran necesidad de contacto constante con sus iguales (Kuss y Griffiths, 2011). Una vez que los jóvenes disponen de un móvil, parecen incapaces de prescindir de él, utilizándolo durante muchas horas, en detrimento de su funcionamiento psicológico y social (Pedrero, Rodríguez y Ruiz, 2012), mostrando mayor vulnerabilidad a su uso excesivo (Ruiz-Olivares, Lucena, Pino y Herruzo, 2010), y mayor propensión a convertirse en un uso problemático o adictivo (Walsh, White y Young, 2008). Los chicos, son más proclives al uso intensivo del móvil con fines lúdicos y funciones tecnológicas destinadas al ocio o entretenimiento (Chóliz y Villanueva, 2011; Chóliz, Villanueva y Chóliz, 2009; Pedrero, Rodrí- guez y Ruiz, 2012). Para ellos el móvil es un elemento que ayuda a mejorar la posición dentro del grupo social (Ling, 2002). Sin embargo, en las chicas el uso del móvil tiene un matiz más emocional (Beranuy, Chamarro, Graner y Carbonell, 2009; Chóliz, Villanueva y Chóliz, 2009; Ling, 2002; Mante y Piris, 2002), relacional y social, mostrando preferencia por los mensajes escritos (Pedrero, Rodríguez y Ruiz, 2012) y pasando más horas hablando por el móvil (Ruiz-Olivares et al., 2010), con el fin de mantener las relaciones interpersonales, fomentar lazos afectivos y favorecer la intimidad en las comunicaciones (Colás, González y de Pablos, 2013; Costa, 2011; De Haro, 2010).

El teléfono móvil está muy presente en los campus universitarios y con frecuencia se utiliza en entornos en los que se produce el aprendizaje (Lepp, Barkley y Karpinski, 2015; Tindell y Bohlander 2012). Además de con fines lúdicos (Barkley y Lepp, 2013), los jóvenes universitarios utilizan el móvil para buscar información sobre sus estudios, comunicarse, organizarse y colaborar con sus compañeros (Organista-Sandoval, McAnally-Salas y Lavigne, 2013). En este sentido, se han apreciado algunas diferencias en la frecuencia o el tipo de uso en función del género, la edad y el campo de conocimiento de los estudiantes universitarios (Ruiz-Olivares et al., 2010), por lo que el estudio de las diferencias en las repercusiones emocionales y comunicacionales del abuso del móvil, en función de estas variables es relevante.

Actualmente, el auge exponencial en el uso de las TIC, así como el dominio y familiaridad de nuestros jóvenes con estas tecnologías (generación interactiva), ha provocado que las formas tradicionales de maltrato entre iguales cambien con el transcurrir del tiempo, apareciendo manifestaciones más específicas que se sirven de las TIC para acosar a la víctima (Smith, Mahdavi, Carvalho y Tippett, 2006). Esta nueva forma de maltrato, denominada de forma general ciberacoso o cyberbullying cuando los implicados son menores, consistiría en una agresión intencional y repetida, por parte de un grupo o un individuo, utilizando recurrentemente formas digitales de contacto social (Internet, telefonía móvil y videojuegos online, principalmente) sobre una víctima, que no puede defenderse por sí sola (León, Felipe, Fajardo y Gómez, 2012).

El ciberacoso es una variación de los tipos convencionales de maltrato: físico, verbal y social-relacional, pero también presenta unas características particulares que lo diferencian (Heirman y Walrave, 2009; Li, 2008; Ortega, Calmaestra y Mora-Merchan, 2008; Slonge y Smith, 2008; Ybarra y Mitchell, 2004). En el ciberacoso no existen lugares donde estar seguros, lo que desarrolla mayor inseguridad en la víctima; el hecho de que el acoso pueda llegar incluso a tu propia casa, provoca sentimientos de indefensión y desprotección. El acoso se hace público y puede ser observado indefinidamente por una mayoría de espectadores. La fuerza física o el tamaño no afectan, ya que el acosador digital, no 
tiene que ser más fuerte que sus víctimas. Por último, existe un desconocimiento y anonimato de las personas agresoras, que provoca en las victimas sentimientos de impotencia.

El uso cada vez mayor del móvil y la sobreexposición a las redes sociales, llaman la atención y provocan alarma social, en especial al constatarse como jóvenes y adolescentes, están expuestos al ciberacoso (Gámez-Guadix, Orue, Smith y Calvete, 2013) a ejercerlo o sufrirlo. El móvil es el dispositivo más utilizado para acosar, hostigar e intimidar a los otros, de manera deliberada y repetitiva (Del Río, Bringué, Sádaba y González, 2009). Sádaba y Bringué (2010) la conciben como la "pantalla que nunca se apaga". Llevar encima el móvil, constantemente, supone poder participar en el acoso a otros, en cualquier momento y lugar, y estar expuesto al mismo, de forma ininterrumpida, durante la mayor parte del día. Un estudio realizado por Giménez, Maquilón y Arnaiz (2015) manifiesta que el acceso a internet a través de móvil, está asociado de forma significativa con la participación en episodios de ciberacoso. En concreto, aportan que el 94,2\% de los ciberagresores poseen un teléfono móvil propio, y el $80,2 \%$ accede a internet a través de él, frente a un 94,7\% de las cibervíctimas. Entre las aplicaciones tecnológicas del móvil que emplean de forma prioritaria, tanto unos como otras, se encuentran el "WhatsApp", las llamadas de voz (Giménez, Maquilón y Arnaiz, 2014) y las redes sociales.

La etapa universitaria es un periodo de transición que en muchos casos conlleva la independencia del núcleo familiar, estrés ante la nueva situación o búsqueda de nuevas amistades, circunstancias que pueden llevar a un cambio en los usos de internet (Fernández-Villa et al., 2015), todo esto, junto con la exposición de los jóvenes al ciberacoso, convierte a los universitarios en una población de especial interés para el estudio de los comportamientos relacionados con el ciberacoso.

En este trabajo se pretende estudiar las repercusiones sociales, personales y comunicacionales del abuso del móvil -en función del género, la edad, el campo de conocimiento y el número de horas de uso del móvil- de los estudiantes universitarios, y profundizar en los diferentes perfiles del ciberacoso, analizando quién presenta más problemas personales y sociales con el uso del móvil ¿`íctimas o agresores? También si el número de horas de uso del móvil tiene un efecto sobre dichos problemas, entendiendo que el acoso tecnológico tiene efectos negativos sobre las víctimas y agresores a nivel, emocional, académico y psicosocial.

\section{Método}

\section{Participantes}

El criterio de inclusión de los participantes fue estar matriculado en la Universidad de Extremadura en el curso 2014-2015. La muestra de participantes estuvo consti- tuida por 1200 estudiantes. La edad media fue de 20,95 años (DT= 3,430; rango 18-32); 58,4\% (n=700) mujeres y el $41,6 \%(n=500)$ varones. Los estudiantes cursaban $1^{\circ}$ $(50,9 \%), 2^{\circ}(25 \%), 3^{\circ}(17,5 \%)$ y $4^{\circ}(5,8 \%)$ curso de diferentes Grados de la Universidad de Extremadura. El número de participantes se determinó a partir del número de estudiantes matriculados en el curso 2014-2015, considerando un error muestral del $3 \%$ y un nivel de confianza del 95,5\%. La selección de los estudiantes se realizó mediante un muestreo polietápico por conglomerados y selección aleatoria de Grado y curso de los estudiantes, en las Facultades de la Universidad de Extremadura. No todos los estudiantes informaron sobre su edad y el campo de conocimiento científico, por lo que en las comparaciones realizadas en relación con la edad y el campo de conocimiento, se perdieron 120 y 88 casos respectivamente.

\section{Instrumentos de Medida}

Cuestionario Sociodemográfico elaborado de forma específica para la investigación, y que contenía cuestiones relativas a edad, género, campo de conocimiento científico de los estudiantes y percepción subjetiva de horas de utilización al día del móvil.

Escala de Victimización a través del Teléfono Móvil, CYBVIC (Buelga, Cava y Musitu, 2010). Esta escala pretende determinar el número de victimizaciones sufridas durante el último año a través del teléfono móvil, que se producen en un contexto determinado. La escala de victimización consta de 10 ítems que evalúan comportamientos que implican agresiones de: 1) Hostigamiento "Me han insultado o ridiculizado con mensajes o llamadas"; 2) persecución "Me han amenazado para meterme miedo"; 3) denigración "Han contado mentiras o rumores falsos sobre mí"; 4) violación de la intimidad "Han compartido mis secretos con otros"; 5) exclusión social "Me han llamado/Me han dicho de conectarme/ y no han contestado"; 6) suplantación de la identidad "Se han hecho pasar por mí para decir o hacer cosas malas en Internet”. El rango de respuesta es de tipo Likert con cuatro opciones de respuesta que oscilan del 1 al 4 (1=Nunca; 2=Pocas veces; $3=$ Muchas veces; 4=Siempre). El coeficiente de fiabilidad alfa de Cronbach $(\alpha)$ que se obtuvo en la validación de la escala de Buelga, Cava y Musitu (2012), fue de 0,85. Con nuestros participantes los índices fueron los siguientes: $\alpha=0,75$, fiabilidad compuesta $(\mathrm{FC})=0,79$, con una varianza media extractada (VME) de 0,50. Los índices indican una adecuada fiabilidad global.

Escala de Agresión a través del Teléfono Móvil, CYB-AGRES (Buelga y Pons, 2012). Esta escala pretende determinar el número de ciberagresiones cometidas durante el último año a través del teléfono móvil. La escala está formada por 10 
ítems que evalúan comportamientos que implican agresiones de: 1) hostigamiento "He insultado o ridiculizado con mensajes o llamadas"; 2) persecución "He amenazado para meter miedo", 3) denigración "He contado mentiras o rumores falsos sobre alguien", 4) violación de la intimidad "He contado secretos de otro para fastidiarle"; 5) exclusión social "He hecho llamadas y no he contestado o he dicho de conectarse y no he respondido" 6) suplantación de la identidad "Me he hecho pasar por otro para decir o hacer cosas malas por el móvil o en internet”. La escala de respuesta es de tipo Likert con cuatro opciones de respuesta (nunca, pocas veces, muchas veces y siempre). El coeficiente de fiabilidad $\alpha$ de la escala, obtenido por Buelga y Pons (2012) en su estudio fue de 0,88. Con nuestros participantes, las puntuaciones obtuvieron un $\alpha$ de 0,82 , $\mathrm{FC}=0,85$, con una VME de 0,52 .

Cuestionario de Experiencias relacionadas con el Móvil, CERM (Beranuy et al., 2009). Con este cuestionario se pretende examinar el grado de "adicción” al teléfono móvil, entre los participantes del estudio. Este cuestionario también cuenta con 10 ítems de tipo Likert con cuatro respuestas, que oscilan del uno al cuatro, en orden creciente de intensidad (1=Nada, 2=Poco, 3=Algo y 4=Bastante). Consta de dos factores: los conflictos y el uso comunicativo/emocional. El factor "Conflictos" se refiere a las repercusiones personales y sociales del abuso del móvil (Beard y Wolf, 2001; Young, 2007): “¿Has tenido el riesgo de perder una relación importante, un trabajo o una oportunidad académica por el uso del móvil?”. El factor “Uso Comunicacional y Emocional" evalúa las repercusiones comunicacionales y emocionales derivadas del uso abusivo del móvil: “¿Piensas que la vida sin móvil es aburrida, vacía y triste?”.

La escala presenta una buena fiabilidad, con un $\alpha$ de .805 (Beranuy et al., 2009). En nuestros participantes, las puntuaciones obtuvieron una puntuación $\alpha$ de 0,80 , FC $=0,80$, con una VME de 0,50. Asimismo, las dimensiones o factores del cuestionario presentan una aceptable fiabilidad y VME $\geq 0,50$ [Conflictos $(\alpha=0,67, \mathrm{FC}=0,78, \mathrm{VME}$ $=0,50)$; Uso Comunicativo y Emocional $(\alpha=0,72, \mathrm{FC}=0,80$, $\mathrm{VME}=0,50)]$.

\section{Procedimiento}

Los diferentes instrumentos fueron aplicados a los estudiantes durante el curso académico 2014/2015. Se siguieron las directrices éticas de la American Psychological Association (APA, 2009) con respecto al consentimiento informado de los participantes, a pesar de ser una investigación que no provoca perjuicio o daño, al tratarse del estudio de métodos de manejo del aula, en un contexto educativo. Se garantizó la confidencialidad de los datos obtenidos y su utilización exclusiva para fines de investigación. La cumplimentación de los cuestionarios se llevó a cabo en el contexto del aula y bajo la presencia del in- vestigador, entrenado para resolver cualquier duda que pudieran llegar a tener, en relación a las preguntas. No se les ofreció compensación, siendo totalmente voluntaria su participación. Los instrumentos se completaron con una duración de entre 20 y 25 minutos.

\section{Análisis de datos}

Las técnicas de análisis de datos utilizadas han sido de corte cuantitativo, aplicándose técnicas de estadística ( $t$ de Student, ANOVA y MANOVA) a través del paquete estadístico SPSS (versión 21). Se somete a los datos a las pruebas de Kolmogorov-Smirnov, Rachas y Levene, encontrándose $p>0,05$ en todas las pruebas, contrastándose así los supuestos de normalidad, aleatorización y homoscedasticidad respectivamente, quedando justificada la utilización de pruebas paramétricas.

\section{Resultados}

Abuso del móvil y diferencias por género, edad y campo de conocimiento científico de los estudiantes.

En la Tabla 1 se pueden observar los resultados de la prueba $t$ de student. Se han encontrado diferencias significativas en el factor "Uso Comunicacional y Emocional", asociada a la variable género de los estudiantes. Las chicas manifiestan más problemas comunicacionales y emocionales por el uso del móvil, que los chicos $(t=6,160, p<$ 0,001, $r=0,18)$.

La prueba ANOVA (Tabla 2), encuentra diferencias significativas entre las puntuaciones medias de los grupos de edad en los dos factores. La prueba de ajuste para comparaciones múltiples de Bonferroni muestra que las diferencias encontradas en el primer factor "Conflictos", sólo son significativas entre el grupo de 18 a 20 años, con el grupo de más de 25 años $(p=0,008)$. Respecto al segundo factor "Uso comunicacional y Emocional", las diferencias son significativas entre el grupo más joven (de 18 a 20 años), con el resto de grupos $p=0,001$, con el grupo de 21 a 24 años $p$ $<0,001$ y con el grupo de más de 25 años.

En la Tabla 3 se presentan los datos de la prueba ANOVA, y se observa que existen diferencias estadísticamente significativas según el campo científico de los estudiantes en la puntuación del factor "Uso Comunicacional y Emocional". Los estudiantes del campo de conocimiento Científico Técnico obtienen puntuaciones más bajas, que el resto de estudiantes.

La prueba de ajuste para comparaciones múltiples de Bonferroni muestra que no existen diferencias significativas entre los campos científicos: Ciencias de la Salud, Ciencias Sociales y Jurídicas y Humanidades. Sin embargo, se han encontrado diferencias significativas entre el campo de conocimiento Científico Técnico y los demás campos científicos: $p<0,001$, con el campo Ciencias de la 
Tabla 1. Diferencias de género en las experiencias relacionadas con el móvil.

\begin{tabular}{lccccccc}
\hline \multirow{2}{*}{ Factores CERM } & \multicolumn{2}{c}{ Varón $(\mathrm{n}=500)$} & \multicolumn{2}{c}{ Mujer $(\mathrm{n}=700)$} & \multicolumn{3}{c}{$t$ de Student } \\
\cline { 2 - 8 } & $M$ & $D T$ & $M$ & $D T$ & $t$ & $p$ & $r$ \\
\hline Conflictos & 6,79 & 2,36 & 6,66 & 1,94 &,- 946 & 0,344 & 0,03 \\
Uso Comunicacional y Emocional & 9,42 & 2,94 & 10,50 & 2,84 & 6,16 & 0,000 & 0,18 \\
\hline
\end{tabular}

Tabla 2. Diferencias en grupos de edad en las experiencias relacionadas con el móvil.

\begin{tabular}{|c|c|c|c|c|c|c|c|c|}
\hline \multirow{3}{*}{ Factores CERM } & \multicolumn{8}{|c|}{ Intervalos de edad } \\
\hline & \multicolumn{2}{|c|}{18 a 20 años $(n=623)$} & \multicolumn{2}{|c|}{21 a 24 años $(n=354)$} & \multicolumn{2}{|c|}{25 a 32 años $(n=103)$} & \multicolumn{2}{|c|}{ ANOVA } \\
\hline & $M$ & $D T$ & $M$ & $D T$ & $M$ & $D T$ & F & $p$ \\
\hline Conflictos & 6,85 & 2,14 & 6,63 & 2,12 & 6,17 & 1,77 & 4,95 & 0,007 \\
\hline $\begin{array}{l}\text { Uso comunicacional } \\
\text { y emocional }\end{array}$ & 10,44 & 2,93 & 9,72 & 2,84 & 8,95 & 2,79 & 15,51 & 0,000 \\
\hline
\end{tabular}

Tabla 3. Diferencias entre campos de conocimiento en las experiencias relacionadas con el móvil.

\begin{tabular}{|c|c|c|c|c|c|c|c|c|c|c|}
\hline & \multicolumn{10}{|c|}{ Campos de conocimiento } \\
\hline & \multicolumn{2}{|c|}{$C S(n=246)$} & \multicolumn{2}{|c|}{ CSJ $(n=550)$} & \multicolumn{2}{|c|}{$\mathrm{CT}(\mathrm{n}=232)$} & \multicolumn{2}{|c|}{$H(n=84)$} & \multicolumn{2}{|c|}{ ANOVA } \\
\hline & $M$ & $D T$ & $M$ & $D T$ & $M$ & $D T$ & $M$ & $D T$ & $F$ & $p$ \\
\hline Conflictos & 6,84 & 2,05 & 6,80 & 2,12 & 6,48 & 2,20 & 10,28 & 2,81 & 1,953 & 0,119 \\
\hline $\begin{array}{l}\text { Uso comunicacional } \\
\text { y emocional }\end{array}$ & 10,28 & 2,81 & 10,48 & 2,93 & 8,86 & 2,64 & 9,95 & 3,23 & 17,94 & 0,000 \\
\hline
\end{tabular}

Nota. $C S=$ Ciencias de la Salud; $C S J=$ Ciencias Sociales y Jurídicas; $C T=C i e n t i ́ f i c o$ Técnico; H=Humanístico

Tabla 4. Medias y desviaciones típicas de los factores "Conflictos" y "Uso Comunicacional y Emocional" por horas de utilización del móvil y perfiles de acoso con el móvil.

\begin{tabular}{|c|c|c|c|c|c|c|c|c|c|}
\hline \multirow{3}{*}{ Factores CERM } & \multirow{3}{*}{$\begin{array}{l}\text { Horas de uso } \\
\text { del móvil al día }\end{array}$} & \multicolumn{8}{|c|}{ Perfiles de acoso } \\
\hline & & \multicolumn{2}{|c|}{ Víctima } & \multicolumn{2}{|c|}{ Agresor } & \multicolumn{2}{|c|}{ Víctima/agresor } & \multicolumn{2}{|c|}{ Sin perfil } \\
\hline & & $M$ & $D T$ & $M$ & $D T$ & $M$ & $D T$ & $M$ & $D T$ \\
\hline \multirow{4}{*}{ Conflictos } & hasta 2 horas & 5,94 & 1,21 & 6,10 & 1,63 & 7,34 & 3,70 & 5,65 & 1,21 \\
\hline & de 3 a 5 horas & 6,78 & 2,36 & 7,00 & 2,39 & 8,16 & 2,42 & 6,21 & 1,59 \\
\hline & de 6 a 10 horas & 6,91 & 1,75 & 7,23 & 1,64 & 7,98 & 2,31 & 6,46 & 2,02 \\
\hline & 11 o más horas & 9,06 & 2,33 & 7,25 & 1,68 & 8,07 & 2,92 & 6,74 & 1,69 \\
\hline \multirow{4}{*}{$\begin{array}{l}\text { Uso } \\
\text { comunicacional } \\
\text { y emocional }\end{array}$} & hasta 2 horas & 7,89 & 2,05 & 8,95 & 2,36 & 10,50 & 3,63 & 7,70 & 2,12 \\
\hline & de 3 a 5 horas & 10,25 & 3,26 & 10,4 & 2,66 & 11,50 & 2,44 & 9,82 & 2,75 \\
\hline & de 6 a 10 horas & 11,20 & 2,74 & 10,85 & 2,63 & 11,70 & 2,10 & 10,20 & 2,50 \\
\hline & 11 o más horas & 12,47 & 3,30 & 12,77 & 2,63 & 12,70 & 2,34 & 10,90 & 2,63 \\
\hline
\end{tabular}

Salud; $p<0,001$, con el campo Ciencias Sociales y Jurídicas; $p=0,017$ y con el campo Humanístico.

Abuso del móvil y perfiles de víctima y agresor.

Para seleccionar aquellos estudiantes que hubiesen experimentado en un mayor número e intensidad los roles de víctima y agresor, se calculó el valor de percentil 75 en las puntuaciones de la Escala de Victimización a través del Teléfono Móvil (CYB-VI) y de la Escala de Agresión a través del Teléfono Móvil (CYB-AGRES). En estas escalas se preguntaba a los estudiantes por su participación, según el número de victimizaciones sufridas durante el último año a través del teléfono móvil o las ciberagresiones cometidas.

Las puntuaciones descriptivas y de percentiles para cada uno de los perfiles fueron los siguientes: víctimas $(M$ = 12,69, $D T=2,75$, percentil 75: 14$)$, agresores $(M=11,67$, $D T=2,99$, percentil 75: 12). Se seleccionaron subgrupos dentro de la muestra final, y se encontraron que había estudiantes que se podían incluir en varias combinaciones posibles dentro de estos roles, de forma que finalmente se establecieron los subgrupos de perfiles: víctimas $(n=110)$, 
agresores $(n=184)$, víctima/agresores $(n=217)$ y $\sin$ perfil $(n=605)$.

A continuación, se examinó la posible influencia del perfil asumido por el estudiante en situación de acoso con el móvil, y las horas de utilización del móvil sobre la puntuación en los factores del Cuestionario de Experiencias relacionadas con el Móvil, CERM. Se realizó un análisis multivariado de la varianza (MANOVA) utilizando como factores fijos, las horas de utilización del móvil (hasta dos horas al día, entre tres y cinco horas, de seis a diez horas y más de once horas) y los cuatro perfiles definidos (víctimas, agresores, víctimas/agresores y sin perfil), y como variables dependientes los dos factores "Conflictos" y "Uso Comunicacional y Emocional" del Cuestionario de Experiencias relacionadas con el Móvil, CERM. Se utilizó el valor de Lambda de Wilks $(\lambda)$ para observar la existencia de diferencias significativas, y como índice de tamaño del efecto, el valor de eta cuadrado parcial $\left(\mathrm{h}^{2}\right)$. En la Tabla 4 se pueden ver los valores de media y desviación típica de los factores "Conflictos" y "Uso Comunicacional y Emocional" por horas de utilización del móvil y perfiles del acoso con el móvil.

Los resultados obtenidos indican diferencias significativas en función de los perfiles asumidos (Wilks $\lambda=0,894$, $\left.p<0,001, \eta^{2}=0,055\right)$, en función de las horas de utilización del móvil (Wilks $\lambda=0,891, p<0,001, \mathrm{n}^{2}=0,056$ ), y en la interacción entre los perfiles asumidos/horas, de utilización del móvil (Wilks $\lambda=0,967, p=0,013, \eta^{2}=0,017$ ).

En relación con los perfiles asumidos, se encontraron diferencias estadísticamente significativas en los dos factores "Conflictos" $\left(F(3,1200)=29,60, p<0,001, \eta^{2}=0,081\right)$ y "Uso Comunicacional y Emocional" $(F(3,1200)=25,57, p<$ $\left.0,001, \eta^{2}=0,071\right)$, en el sentido de una puntuación media superior en los estudiantes con un perfil víctima/agresor y agresor (ver Tabla 4). Para el factor "Conflictos", las comparaciones múltiples de Bonferroni muestran que las mayores diferencias significativas aparecen entre los subgrupos víctima/agresor, seguido por el perfil víctima, en comparación con los participantes sin perfil o no involucrados en el acoso con móvil. Respecto al factor "Uso Comunicacional y Emocional" las mayores diferencias significativas aparecen entre los subgrupos víctima/agresor, seguido por el perfil agresor, en comparación con los participantes sin perfil. Existen diferencias en ambos factores, entre todos los pares de comparaciones de los diferentes perfiles, excepto en el perfil víctima con el perfil agresor.

En cuanto a las horas de utilización del móvil, también los resultados ponen de manifiesto, diferencias estadísticamente significativas en los dos factores "Conflictos" ( $F(3$, $\left.1200)=12,21, p<0,001, \eta^{2}=0,035\right)$ y "Uso Comunicacional y Emocional" $\left(F(3,1200)=40,16, p<0,001, \eta^{2}=0,107\right)$. Las comparaciones múltiples de Bonferroni muestran para el factor "Conflictos", que las mayores diferencias significativas aparecen entre los estudiantes que utilizan más de once horas al día el móvil, y los que lo utilizan dos horas.
No se encuentran diferencias entre el subgrupo más de once horas y el subgrupo de seis a diez horas. Para el factor "Uso Comunicacional y Emocional" las mayores diferencias significativas se dan entre el subgrupo más de once horas, en comparación con el subgrupo dos horas. Se encuentran diferencias significativas entre todos los subgrupos, puntuaciones medias superiores en los estudiantes con más horas de uso diario del móvil.

Por último, respecto a la interacción entre los perfiles asumidos/horas de utilización del móvil, sólo se encontraron diferencias estadísticamente significativas en el factor "Conflictos" $\left(F(9,1200)=2,083, p<0,028, \eta^{2}=0,018\right)$. Hallando diferencias significativas entre los estudiantes que utilizan más de once horas al día el móvil, y los que lo utilizan hasta dos horas, en los perfiles: víctima, agresor y sin perfil, no existiendo una interacción significativa en el perfil víctima/agresor.

\section{Discusión}

El teléfono móvil se ha convertido en la tecnología más popular entre los jóvenes, y en una herramienta imprescindible en su vida diaria, provocando en ocasiones, cierto uso problemático o adictivo (Chóliz, 2012), siendo el dispositivo más utilizado para acosar, hostigar e intimidar a los otros (Del Río, et al., 2009). De acuerdo con esto, el propósito de este trabajo fue analizar las repercusiones sociales, personales y comunicacionales del abuso del móvil de los estudiantes universitarios, y profundizar en los diferentes perfiles del ciberacoso.

Abuso del móvil y diferencias por género, edad y campo de conocimiento científico de los estudiantes

En cuanto al género, el uso abusivo del móvil genera conflictos en los jóvenes de ambos sexos por igual, y las chicas manifiestan más problemas comunicacionales y emocionales, que los chicos. Estos resultados coinciden con otros estudios, que afirman que las mujeres tienen más probabilidades que los varones, de presentar consecuencias negativas del uso desadaptativo del móvil (Beranuy, Oberst, Carbonell y Chamarro, 2009; Takao, Takahashi y Kitamura, 2009). El móvil permite una conexión afectiva con los otros (Aguado y Martínez, 2006), lo que provoca un aumento en su uso, especialmente en las chicas (Sánchez-Martínez y Otero, 2009), que lo usan para mantener dicha aproximación afectiva, y para hacer frente a estados emocionales desagradables (Chóliz, Villanueva y Chóliz, 2009).

En relación a la variable edad, los resultados confirman que a menor edad (18 a 20 años) surgen mayores conflictos comunicacionales y emocionales por el uso del móvil, que en edades superiores (grupo de 21 a 24 años, y grupo de más de 25 años). Resultados coincidentes, con un estudio realizado por De la Villa y Suárez (2016) quienes conclu- 
yen que tanto los problemas relacionados con el uso emocional y comunicativo del móvil, como los conflictos que genera dicho uso, se incrementan durante la adolescencia media, con respecto a la preadolescencia. Así, los más jóvenes, aquellos que están saliendo de la adolescencia (18 a 20 años) ven el móvil como algo natural. Mientras, los jóvenes "mayores" (grupo de 21 a 24 años y grupo de más de 25 años) usan el móvil con fines más profesionales, menos lúdicos y con menos consecuencias negativas (Beranuy, Chamarro, Graner y Carbonell, 2009), como así lo confirman ciertos estudios (Derbyshire et al., 2013), puesto que se eleva en ellos la percepción del problema que supone el tiempo excesivo dedicado al uso del móvil y sus posibles consecuencias adversas (Labrador y Villadangos, 2010).

La edad es por tanto, una variable relevante que está condicionando la forma de comportarse y de relacionarse socialmente, ante el uso del móvil. Cada vez es más temprana la edad del primer acceso a dicha tecnología, y la disposición de teléfono móvil se incrementa significativamente a partir de los 10 años, hasta alcanzar el 100\% a partir de los 17 años (INE, 2015). Cuanto más jóvenes son los usuarios, mayor es el porcentaje de personas con un índice elevado de uso problemático del móvil. La prevalencia en los adolescentes españoles (12 y 18 años) se sitúa entre un 15-20\% (Flores, Jenaro, González, Martín y Poy, 2013; Jenaro, Flores, Gómez-Vela, González-Gil y Caballo, 2007; Labrador y Villadangos, 2010; López-Fernández, Honrubia-Serrano y Freixa-Blanxart, 2012; Sánchez-Martínez y Otero, 2009) y en la población universitaria en un 7,99\% (Jenaro et al., 2007). Estos resultados relacionados con la edad -más allá del uso del móvil- tienen relación con la etapa evolutiva de la adolescencia, caracterizada por poca experiencia de vida, dificultad para reconocer adicciones sutiles y la sensación de normalidad ante conductas de riesgo (Castellana, Sánchez-Carbonell, Granery Beranuy, 2007), así como déficit para postergar, planificar y considerar consecuencias futuras (Corona y Peralta, 2011). Es una etapa en la que los adolescentes son más fácilmente influenciables y presentan un menor control de los impulsos (Muñoz-Rivas y Agustín, 2005).

Según el campo científico de los estudiantes en cuanto al "Uso Comunicacional y Emocional" del móvil, los estudiantes del campo de conocimiento Científico Técnico obtienen puntuaciones más bajas que el resto de estudiantes (Ciencias de la Salud, Ciencias Sociales y Jurídicas y Humanidades). Dos posibles hipótesis pueden ayudar a interpretar estos resultados, por un lado la dificultad de las carreras técnicas, y por otro el sesgo del género. Con relación a la primera cuestión, en el curso 2014-15 en los grados del campo científico técnico, el número medio de créditos superados, la tasa de rendimiento (relación entre créditos superados y créditos matriculados) y la nota media del expediente académico es la más baja en comparación con el resto de campos científicos (Ministerio de Educación, Cultura y Deporte, 2015).Los estudiantes perciben como más difíciles las carreras técnicas, y más fáciles las de ciencias sociales. Una mayor exigencia en las carreras técnicas, sin duda, condiciona el uso del móvil de los estudiantes que las cursan, utilizándolo más para tareas académicas, como por ejemplo: acceso a la agenda de actividades de las diferentes asignaturas a través del Campus Virtual, acceso a los servicios de la biblioteca digital, a la plataforma universitaria "webmail" de correo electrónico..., en detrimento de un uso intensivo con fines lúdicos, comunicacionales y sociales.

Respecto al sesgo del género, en el curso 2014-15 el porcentaje de varones matriculados en grados del campo científico Técnico era de 74,1\%, frente a un 25,9\% de mujeres. En el resto de campos, el porcentaje de mujeres es superior al de varones. En nuestra muestra en el campo científico Técnico, el $72 \%$ son varones frente a un $28 \%$ de mujeres. Como apuntábamos anteriormente cuando analizamos la variable género, nuestros resultados manifiestan que las chicas tienen más problemas comunicacionales y emocionales, que los chicos. Las diferencias encontradas en el factor "Uso Comunicacional y Emocional" entre los estudiantes de campo de conocimiento Científico Técnico y el resto de campos, podría deberse al desequilibrio entre el número de varones y mujeres.

\section{Abuso del móvil y perfiles de víctima y agresor}

Según los resultados obtenidos, se encuentra que el perfil víctima/agresor, es el subgrupo que tiene más conflictos con el uso del móvil, seguido por el perfil víctima. Tanto un perfil como otro, presentan como conducta característica, estar pendiente de los mensajes que reciben constantemente, con comentarios amenazantes y de hostigamiento, que los otros hacen sobre ellos, a través del móvil (Li, 2008; Mason, 2008; Slonje y Smith, 2008). Esto podría derivar en un aumento de conflictos personales e interpersonales. En este sentido, ciertos estudios concluyen que el ciberacoso genera sobre víctima y víctima/agresor, sentimientos de ansiedad, depresión, baja autoestima, irritabilidad y trastornos del sueño (Garaigordobil, 2011), que revierten en un uso excesivo del móvil y un mayor uso problemático de Internet (Ehrenberg, Juckes, White y Walsh, 2008; Gámez-Guadix et al., 2013). De esta manera, tanto víctimas, como víctimas-agresores modifican sus hábitos sociales, laborales y/o académicos, suelen aislarse, y el móvil se convierte en un refugio para ellos, desde donde buscar relaciones virtuales más reforzantes, y como compensación social a las relaciones cara a cara con sus amigos (García del Castillo et al., 2008; Giménez et al., 2015; Kuss y Griffiths, 2011).

Respecto al Uso Comunicacional y Emocional son los subgrupos víctima/agresor, seguido por el perfil agresor, los que presentan mayores repercusiones comunicacionales (aumento de conductas de agresividad, menor control de 
impulsos) y emocionales (inquietud, ansiedad, enfado e irritación), derivadas del uso abusivo del móvil, en comparación con los participantes sin perfil. Estos resultados son coincidentes con otros estudios (Garaigordobil, 2011; Giménez et al., 2015) que confirman la existencia de conductas de agresividad, cambio de intereses y niveles altos de ansiedad, entre tales participantes de ciberacoso.

\section{Horas de utilización del móvil}

En cuanto a las horas de utilización del móvil, los resultados ponen de manifiesto que aquellos estudiantes que utilizan el móvil más de once horas al día, tienen más conflictos con su uso, y este "uso" tiene un matiz más comunicacional y emocional, que aquellos que lo utilizan dos horas .Según Echeburúa y de Corral (2010) las personas pueden hablar por el móvil por la utilidad o el placer de la conducta en sí misma, mientras que una persona adicta, lo hace buscando el alivio del malestar emocional (aburrimiento, soledad, ira, nerviosismo,...). En este sentido, ciertos estudios concluyen que son las personas agresoras, quienes superan a las personas víctimas y a los no implicados, en el consumo diario del móvil (Giménez et al., 2015), con el riesgo de convertirse en una adicción, máxime si tenemos en cuenta que los estudiantes suelen subestimar el uso diario del móvil, aseverando dedicar entre una y cuatro horas, cuando la realidad confirma que son bastantes más (Aslanidou y Menexes, 2008; Garmendia, Garitaonandia, Martínez y Casado, 2012; Hunley et al., 2005).

Por último, respecto a la interacción entre los perfiles asumidos/horas de utilización del móvil (víctima, agresor y sin perfil) se encuentran con mayores conflictos, aquellos que utilizan el móvil más de once horas al día, frente a los que lo utilizan hasta dos horas, no ocurriendo esto en el caso del perfil víctima/agresor, en el que el número de horas no determina los conflictos. En este sentido, se puede pensar que las personas víctimas cuando responden con agresión, manifiestan con más intensidad los problemas intrapersonales e interpersonales derivados del uso del móvil. Diversas investigaciones demuestran que los sujetos que asumen el rol complejo de víctima/agresor, informan de mayor nivel sintomatológico y un mayor índice de distrés, en comparación con el resto de perfiles de bullying (Felipe, León y Fajardo, 2013; Haynie et al., 2001; Kaltiala-Heino, Rimpela, Rantanen y Rimpela, 2000; Kim, Leventhal, Koh, Hubbard y Boyce, 2006; Stein, Dukes y Warren, 2007).

\section{Limitaciones}

El estudio aquí realizado presenta varias limitaciones, como son la utilización de autoinformes, como único método de recogida de información, tanto para la evaluación de las situaciones de ciberacoso, como del abuso del móvil. Además, las diferencias en el número de casos en cada uno de los roles descritos, debe llevar a considerar los resultados de forma cautelosa hasta poder ampliar su número: víctimas $(n=110)$, agresores $(n=184)$, víctima/agresores $(n=217)$ y $\sin$ perfil $(n=605)$. Por otro lado, es importante resaltar que la muestra sólo es representativa de población universitaria, por lo que los resultados obtenidos no pueden generalizarse a población no universitaria.

\section{Conclusiones}

Por un lado, es necesario incidir en la educación en valores de niños, jóvenes y adultos para un uso positivo, no nocivo y responsable de las tecnologías de la comunicación, que cada vez más, están al alcance en cualquier momento y lugar, en muchos casos -principalmente en menores- sin un adecuado control o supervisión, que expone a niños y jóvenes a un gran número de situaciones de riesgo. La escuela tiene la obligación y la oportunidad de generar espacios de convivencia y cambios de actitudes hacia el uso de dichas tecnologías, siendo importante, dotar de recursos al profesorado para prevenir actitudes no deseadas, y afrontar las diferentes modalidades de acoso.

Por otro lado, en la línea de lo anterior, se puede afirmar que es necesario implementar programas de prevención más allá de la escuela y los institutos, por la importancia de erradicar las situaciones de acoso y/o ciberacoso, con el objetivo de que los jóvenes se identifiquen con los valores de respeto, empatía y no violencia, que deben primar en el ámbito universitario. Se debe incidir en la importancia de investigaciones, que busquen la identificación de todas las formas de acoso, poniendo especial énfasis en las que se producen a través de las tecnologías emergentes, profundizando sobre los usos positivos y las consecuencias de su abuso, mediante la promoción del uso responsable y un disfrute saludable, como estrategias preventivas de la violencia digital.

\section{Agradecimientos}

Este estudio ha sido posible gracias a la participación de los estudiantes y profesores de la Universidad de Extremadura.

\section{Conflictos de intereses}

Los autores declaran no tener ningún conflicto de intereses.

\section{Referencias}

Aguado, J. M. y Martínez, I. J. (2006). El proceso de mediatización de la telefonía móvil: de la interacción al consumo cultural. Estudios de Comunicación (ZER), 11, 319-343. 
American Psychological Association (APA) (2009). Report of the Ethics Committee, 2008. American Psychologist, 64, 464-473.

Aslanidou, S. y Menexes, G. (2008). Youth and the internet: Uses and practices in the home. Computers Eै Education, 51, 1375-1391. doi:10.1016/j.compedu.2007.12.003.

Beard, K. W. y Wolf, E. M. (2001). Modification in the proposed diagnostic criteria for Internet addiction. Cyberpsychology E Behavior, 4, 377-383 doi: 10.1089/109493101300210286.

Beranuy, M., Chamarro, A., Graner, C. y Carbonell, X. (2009). Validación de dos escalas breves para evaluar la adicción a internet y el abuso del móvil. Psicothema, 21, 480-485.

Beranuy, M., Oberst, U., Carbonell, X. y Chamarro, A. (2009). Problematic Internet and mobile phone use and clinical symptoms in college students: The role of emotional intelligence. Computers in Human Behavior, 25, 1182-1187. doi:10.1016/j.chb.2009.03.001.

Bianchi, A. y Phillips, J. G. (2005). Psychological Predictors of Problem Mobile Phone Use. Cyberpsychology $\mathcal{E}^{\circ}$ Behavior, 8, 39-51. doi: 10.1089/cpb.2005.8.39.

Buelga, S. y Pons, J. (2012). Agresiones entre Adolescentes a través del Teléfono Móvil y de Internet. Psychosocial Intervention, 21, 91-101. doi:10.5093/in2012v21n1a2.

Buelga, S., Cava, M. J. y Musitu, G. (2010). Cyberbullying: victimización entre adolescentes a través del teléfono móvil y de Internet. Psicothema, 22, 784-789.

Buelga, S., Cava, M. J. y Musitu, G. (2012). Validación de la escala de victimización entre adolescentes a través del teléfono móvil y de internet. Pan American Journal of Public Health 32, 36-42. doi:10.1590/S102049892012000700006.

Castellana, M., Sánchez-Carbonell, X., Graner, C. y Beranuy, M. (2007). El adolescente ante las tecnologías de la información y la comunicación: Internet, móvil y videojuegos. Papeles de Psicólogo, 28, 196-204.

Chóliz, M. (2010). Mobile phone addiction: a point of issue. Addiction, 105, 373-374. doi: 10.1111/j.1360-0443.2 009.02854.x.

Chóliz, M (2012). Mobile-phone addiction in adolescence: The Test of Mobile Phone Dependence (TMD). Progress in Health Sciences, 2, 33-44.

Chóliz, M., Villanueva, V. y Chóliz, M. C. (2009). Ellas, ellos y su móvil: Uso, abuso (¿y dependencia?) del teléfono móvil en la adolescencia. Revista Española de Drogodependencias, 1, 74-88.

Chóliz, M. y Villanueva, V. (2011). Evaluación de la adicción al móvil en la Adolescencia. Revista Española de Drogodependencias, 36, 165-184.

Colás, P., González, T. y de Pablos, J. (2013). Juventud y redes sociales: Motivaciones y usos preferentes. Comunicar, 40, 15-23. doi:10.3916/C40-2013-02-01.
Corona, H. F. y Peralta, V. E. (2011). Prevención de conductas de riesgo. Revista Médica Clínica Las Condes, 22, 68-75. doi: 10.1016/S0716-8640(11)70394-7.

Costa, J. (2011). Los jóvenes portugueses de los 10 a los 19 años: ¿qué hacen con los ordenadores?. Education in the Knowledge Society (EKS), 12, 209-239.

De Haro, J. J. (2010). Redes Sociales para la educación. Madrid: Anaya.

Derbyshire, K. L., Lust, K. A., Schreiber, L. R., Odlaug, B. L., Christenson, G. A., Golden, D. J. y Grant, J. E. (2013). Problematic Internet use and associated risks in a college sample. Comprehensive Psychiatry, 54, 415-422. doi.10.1016/j.comppsych.2012.11.003.

Del Río, J., Bringué, X., Sádaba, C. y González, D. (2008). Cyberbullying: un análisis comparativo en estudiantes de Argentina, Brasil, Chile, Colombia, México, Perú y Venezuela. En V Congrés Internacional Comunicació $i$ Realitat (pp. 307-316). Barcelona, ES: Universitat Ramon Llull.

De la Villa, M. y Suárez, C. (2016). Factores de riesgo en el uso problemático de Internet y del teléfono móvil en adolescentes españoles. Revista Iberoamericana de Psicología y Salud, 7, 69-78. doi:10.1016/j.rips.2016.03.001.

Díaz-Gandasegui, V. (2011). Mitos y realidades de las redes sociales. Información y comunicación en la Sociedad de la Información. Prisma Social, 6, 1-26.

Echeburúa, E. y de Corral, P. (2010). Adicción a las nuevas tecnologías y a las redes sociales en jóvenes: Un nuevo reto. Adicciones, 22, 91-96. doi:10.20882/adicciones.196.

Echeburúa, E., Labrador, F. J. y Becoña, E. (2009). Adicción a las Nuevas Tecnologías. Madrid: Pirámide.

Ellwood-Clayton, B. (2003). Virtual strangers: Young love and texting in the Filipino archipelago of cyberspace. In K. Nyíri (Ed.), Mobile Democracy: Essays on Society, Self, and Politics (pp. 225-239). Vienna, Austria: Passagen Verlag.

Ehrenberg, A., Juckes, S., White, K. M. y Walsh, S. P. (2008). Personality and self-esteem as predictors of young people's technology use. Cyberpsychology $\mathcal{E}^{2}$ Behavior, 11, 739741. doi:10.1089/cpb.2008.0030.

Felipe, E., León, B. y Fajardo, F. (2013). Perfiles psicopatológicos en participantes en situaciones de acoso escolar en Educación Secundaria. Psicología Conductual, 21, 475-490.

Fernández-Villa, T., Alguacil, J., Almaraz, A., Cancela, J., Delgado-Rodríguez, M., García-Martín, M., ... Martín, V. (2015). Uso problemático de internet en estudiantes universitarios: factores asociados y diferencias de género. Adicciones, 27, 265-275. doi:10.20882/adicciones.751.

Flores, N., Jenaro, C., González, F., Martín, E. y Poy, R. (2013). Adicción al móvil en alumnos de secundaria: efectos en la convivencia. European Journal of Investigation in Health, Psychology and Education, 3, 215-225. doi:10.1989/ejihpe.v3i3.44. 
Gámez-Guadix, M., Orue, I., Smith, P. K. y Calvete, E. (2013). Longitudinal and reciprocal relations of cyberbullying with depression, substance use, and problematic internet use among adolescents. Journal of Adolescent Health, 53, 446-452. doi:10.1016/j.jadohealt2013.03.030.

Garaigordobil, M. (2011). Prevalencia y consecuencias del cyberbullying: una revisión. International Journal of Psychology and Psychological Therapy, 11, 233-254.

García del Castillo, J. A., Terol, M. C., Nieto, M., Lledó, A., Sánchez, S., Martín-Aragón, M. y Sitges, E. (2008). Uso y abuso de Internet en jóvenes universitarios. Adicciones, 20, 131-142. doi:10.20882/adicciones.277.

Garmendia, M., Garitaonandia, C., Martínez, G. y Casado, M. A. (2012). Los menores en internet. Usos y seguridad desde una perspectiva europea. Quaderns del CAC, $15,37-544$

Giménez, A. M., Maquilón, J. J. y Arnaiz, P. (2014). Acceso a las tecnologías, rendimiento académico y cyberbullying en escolares de secundaria. Revista Iberoamericana de Psicología y Salud, 5, 119-133.

Giménez, A. Maquilón, J. J. y Arnaiz, P. (2015). Usos problemáticos y agresivos de las TIC por parte de adolescentes implicados en cyberbullying. Revista de Investigación Educativa, 33, 335-351. doi: http://dx.doi.org/10.6018/ rie.-33.2.199841.

Gómez, P., Rial, A., Braña, T., Varela, J. y Barreiro, C. (2014). Evaluation and early detection of problematic Internet use in adolescents. Psicothema, 26, 21-26. doi:10.7334/ psicothema2013.109.

Haynie, D. L., Nansel, T., Eitel, P., Davis-Crump, A., Saylor, K., Yu, K. y Simons-Morton, B. (2001). Bullies, victims, and bully/victims: Distinct groups of atriskyouth. Journal of Early Adolescence, 21, 29-49. doi: 10.1177/0272431601021001002.

Heirman, W. y Walrave, M. (2009). Asseing Issues and Concerns about the Mediation of Technology in Cyberbullying. Trípodos Extra 1, 317-329.

Hunley, S. A., Evans, J. H., Delgado-Hachey, M., Krise, J., Rich, T. y Schell, C. (2005). Adolescent computer use and academic achievement. Adolescence, 40, 307-318.

Instituto Nacional de Estadística [INE] (2014). Encuesta sobre Equipamiento y Uso de Tecnologías de Información y Comunicación en los Hogares (TIC-H). Madrid: Instituto Nacional de Estadística. Recuperado de http://www. ine.es/prensa-/np864.pdf

Instituto Nacional de Estadística [INE] (2015). Encuesta sobre Equipamiento y Uso de Tecnologías de Información y Comunicación en los Hogares (TIC-H). Madrid: Instituto Nacional de Estadística. Recuperado de http://www. ine.es/prensa-/np933.pdf

Jenaro, C., Flores, N., Gómez-Vela, M., González-Gil, F. y Caballo, C. (2007). Problematic Internet and cellphoneuse: Psychological, behavioral, and health co- rrelates. Addiction Research and Theory, 15, 309-320. doi:10.1080/16066350701350247.

Kaltiala-Heino, R., Rimpela, M., Rantanen, P. y Rimpela, A. (2000). Bullying at school: An indicatorof adolescents at risk for mental disorders. Journal of Adolescence, 23, 661-674. doi:10.1006/jado.2000.0351.

Kamibeppu, K. y Sugiura, H. (2005). Impact of the mobile phone on junior high-school students' friendships in the Tokio metropolitan area. Cyberpsychology E Behaviour, 8, 121-130. doi:10.1089/cpb.2005.8.121.

Kim, Y. S., Leventhal, B. L., Koh, Y. J., Hubbard, A. y Boyce, W. T. (2006). School bullying and youth violence: causes or consequences of psychopathologic behavior? Archives of General Psychiatry, 63, 1035-1041. doi:10.1001/ archpsyc.63.9.1035.

Kubey, R. W., Lavin, M. J. y Barrows, J. R. (2001). Internet use and collegiate academic performance decrements: early findings. Journal of Communication, 51, 366-382. doi:10.1111/j.1460-2466.2001.tb02885.x.

Kuss, D. J. y Griffiths, M. D. (2011). Online Social Networking and Addiction - A Review of the Psychological Literature. International Journal of Environmental Research and Public Health, 8, 3528-3552. doi:10.3390/ijerph8093528.

Labrador, F. J. y Villadangos, S. M. (2010). Menores y nuevas tecnologías: conductas indicadoras de posible problema de adicción. Psicothema, 22, 180-188.

Labrador, F. J., Villadangos, S. M., Crespo, M. y Becoña, E. (2013). Design and validation of the new technologies problematic use questionnaire. Anales de Psicología, 29, 836-847.doi:10.6018/analesps.29.3.159291.

León, B., Felipe, E., Fajardo, F. y Gómez, T. (2012). Cyberbullying in a sample of secondary students: modulating variables and social networks. Electronic Journal of Research in Educational Psychology, 10, 771-788.

Lepp, A., Barkley, J. E. y Karpinski, A. C. (2015). The relationship between cell phone use and academic performance in a sample of US college students. SAGE Open, 5, 1-9. doi:10.1177/2158244015573169.

Li, Q. (2008). A cross-cultural comparison of adolescents experience related to cyberbullying. Educational Research, 50, 223-234. doi:10.1080/00131880802309333.

Ling, R. (2002). Chicas adolescentes y jóvenes adultos varones: dos subculturas del teléfono móvil. Estudios de Juventud, 57, 33-46.

López-Fernández, O., Honrubia-Serrano, M. y FreixaBlanxart, M. (2012). Adaptación española del "Mobile Phone Problem Use Scale” para población adolescente. Adicciones, 24, 123-130. doi:10.20882/adicciones.104.

Mante, E. y Piris, D. (2002). El uso de la mensajería móvil por los jóvenes en Holanda. Estudios de Juventud, 57, 4758.

Mason, K. L. (2008). Cyberbullying: A preliminary assessment for school personnel. Psychology in the School, 45, 323-348. doi: 10.1002/pits.20301. 
Ministerio de Educación, Cultura y Deporte (2015). Datos y cifras: Curso escolar 2015/2016. Madrid: Secretaría General Técnica. Subdirección General de Documentación y Publicaciones.

Morahan-Martin, J. y Schumacher, P. (2000). Incidence and correlates of pathological Internet use among college students. Computers in Human Behavior, 16, 13-29. doi: 10.1016/S0747-5632(99)00049-7.

Muñoz-Rivas, M. J. y Agustín, S. (2005). La adicción al teléfono móvil. Psicología Conductual, 13, 481-493.

Organista-Sandoval, J., McAnally-Salas, L. y Lavigne, G. (2013). El teléfono inteligente (smartphone) como herramienta pedagógica. Apertura, 5, 6-19.

Ortega, R., Calmaestra, J. y Mora-Merchán, J. A. (2008). Cyberbullying. International Journal of Psychology and Psychologic al Therapy, 8, 183-192.

Pedrero, E., Rodríguez, M. T. y Ruíz, J. M. (2012). Adicción o abuso del teléfono móvil. Revisión de la literatura. Adicciones, 24, 139-152. doi:10.20882/adicciones.107.

Ruiz-Olivares, R., Lucena, V., Pino, M. y Herruzo, J. (2010). Análisis de comportamientos relacionados con el uso/abuso de Internet, teléfono móvil, compras y juego en estudiantes universitarios. Adicciones, 22, 301-310. doi:10.20882/adicciones.171.

Sádaba, C. y Bringué, X. (2010). Niños y adolescentes espanoles ante las pantallas: Rasgos configuradores de una generación interactiva. CEE Participación Educativa, 15, 86-104.

Sánchez-Martínez, M. y Otero, A. (2009). Factors associated with cell phone use in adolescents in the community of Madrid (Spain). Cyberpsychology E Behavior, 12, 131-137. doi:10.1089/cpb.2008.0164.

Slonje, R. y Smith, P. K. (2008). Cyberbullying: Another main type of bullying?. Scandinavian Journal of Psychology, 49, 147-154. doi:10.1111/j.1467-9450.2007.00611.x.

Smith, P. K., Mahdavi, J., Carvalho, M. y Tippett, N. (2006). An investigation into cyberbullying, its forms, awareness and impact, and the relationship between age and gender in cyberbullying. Research Brief No. RBX03-06. London: DfES

Stein, J. A., Dukes, R. L. y Warren, J. I. (2007). Adolescent male bullies, victims, and bully-victims: A comparison of psychosocial and behavioral characteristics. Journal of Pediatric Psychology, 32, 273-282. doi:10.1093/jpepsy/ js1023.

Takao, M., Takahashi, S. y Kitamura, M. (2009). Addictive personality and problematic mobile phone use. Cyberpsychology $\mathcal{E}$ Behavior, 12, 501-507. doi: $10.1089=$ cpb. 2009.0022

Taylor, A. S. y Harper, R. (2003). The gift of the gab? A design oriented sociology of young people's use of mobiles. Journal of Computer Supported Cooperative Work, 12, 267-296.

Tindell, D. R. y Bohlander, R. W. (2012). The use and abuse of cell phones and text messaging in the classroom:
A survey of college students. College Teaching, 60, 1-9. do i:10.1080/87567555.2011.604802.

Treuer, T., Fabian, Z. y Furedi, J. (2001). Internet addiction associated with features of impulse control disorder: Is it a real psychiatric disorder?. Journal of Affective Disorders, 66, 266-283.

Walsh, S. P., White, K. M. y Young, R. M. (2008). Over-connected? A qualitative exploration of the relationship between Australian youth and their mobile phones. Journal of Adolescence, 31, 77-92. doi: 10.1016/j.adolescence-.2007.04.004.

Weare, K. (2004). What impact is having information technology on our young people's health and well-being?. Health Education, 104, 129-131. doi: 10.1108/09654280410544963.

Ybarra, M. y Mitchell, K. (2004). Youth engaging in online harassment: Associations with caregiver-child relationships, Internet use, and personal characteristics. Journal of Adolescence, 2, 319-336. doi:10.1016/j.adolescence.2004.03.007.

Young, K. (2007). Cognitive behavior therapy with internet addicts: Treatment outcomes and implications. Cyberpsychology E B Behavior, 10, 671-679. doi:10.1089/ cpb.2007.9971. 